\title{
The Building of Galactic Disks: Insights from the Triangulum Spiral Galaxy Messier 33
}

\author{
David L. Block ${ }^{1}$, Ivânio Puerari ${ }^{2}$, Giovanni G. Fazio ${ }^{3}$, Alan
}

Stockton $^{4}$, Gabriela Canalizo ${ }^{5}$, Kenneth C. Freeman ${ }^{6}$, Thomas H. Jarrett $^{7}$, Françoise Combes ${ }^{8}$ Robert Groess $^{1}$, Guy Worthey ${ }^{9}$, Robert D. Gehrz ${ }^{10}$, Charles E. Woodward ${ }^{10}$ and Elisha F. Polomski ${ }^{10}$

${ }^{1}$ Anglo American Cosmic Dust Laboratory, School of Computational and Applied

Mathematics, University of Witwatersrand, Johannesburg, 2050, South Africa email: block@cam.wits.ac.za

${ }^{2}$ Instituto Nacional de Astrofísica, Optica y Electrónica, Tonantzintla, 72840, México email: puerari@inaoep.mx

${ }^{3}$ Harvard-Smithsonian Center for Astrophysics, 60 Garden St., Cambridge, MA 02138, USA email: gfazio@cfa.harvard.edu

${ }^{4}$ Institute for Astronomy, University of Hawaii, 2680 Woodlawn Drive, Honolulu, Hawaii, USA email: stockton@ifa.hawaii.edu

${ }^{5}$ Institute of Geophysics and Planetary Physics and Department of Physics, University of California, Riverside, CA 92521, USA

${ }^{6}$ Mount Stromlo and Siding Spring Observatories, Research School of Astronomy and Astrophysics, Australian National University, Australia email: kcf@mso.anu.edu.au

${ }^{7}$ Infrared Processing and Analysis Centre, 100-22, CALTECH, 770 South Wilson Ave, Pasadena, CA 91125, USA email: jarrett@ipac.caltech.edu

${ }^{8}$ Observatoire de Paris, LERMA, 61 Av. de l'Observatoire, F-75014, Paris, France email: francoise.combes@obspm.fr

${ }^{9}$ Washington State University, 1245 Webster Hall, Pullman, WA 99163-2814, USA email: gworthey@wsu.edu

${ }^{10}$ Department of Astronomy, University of Minnesota, 116 Church St. SE, Minneapolis MN 55455, USA

email:gehrz@astro.umn.edu

\begin{abstract}
The Triangulum Spiral Galaxy Messier 33 offers unique insights into the building of a galactic disk. We identify spectacular arcs of intermediate age (0.6 Gyr -2 Gyr) stars in the low-metallicity outer disk. The northern arc spans $\sim 120$ degrees in azimuth and up to 5 arcmin in width. The arcs are located 2-3 disk scale lengths from the galaxy centre (where 1 disk scale length is equivalent to 0.1 degrees in the V-band) and lie precisely where there is a warp in the HI profile of M33. Warps and infall are inextricably linked (Binney, 1992). We present spectroscopy of candidate stars in the outer northern arc, secured using the Keck I telescope in Hawaii. The target stars have estimated visual magnitudes as faint as $\mathrm{V} \sim 25 \mathrm{~m}$. Absorption bands of $\mathrm{CN}$ are seen in all spectra reported in this review talk, confirming their carbon star status. Also presented are PAH emissivity radial profiles generated from IRAC observations of M33 using the Spitzer Space Telescope. A dramatic change of phase in the $m=2$ Fourier component is detected at the domain of the arcs. M33 serves as an excellent example how the disks of spiral galaxies in our Universe are built: as dynamically open systems, growing from the inward, outward.
\end{abstract}

Keywords. galaxies: evolution - galaxies: spiral - galaxies: individual (M33 = NGC598) 


\section{Introduction}

Spiral galaxies appear to be dynamically open systems, whose disks are still forming at the current epoch and which continue to accrete mass. Isolated spiral galaxies (non-accreting systems) cannot reproduce the observed distribution of gravitational bar torques at all (see Figure 1 in Block et al. 2002). In that study, it was argued that disk masses should double every 10 billion years. The most likely source for the origin of accreted gas could be the reservoirs of gas observed outside nearly all spiral disks (Sancisi 1983). Keres et al. (2005) carefully discuss accretion in terms of filaments in the cosmic web. An extremely important point is that accreting systems in gas need not show any signs of accretion in stars, such as the presence of tidal tails, stellar loops or close companions. Disks of galaxies appear to form from the inside out (Block et al. 2002). Relative to the inner regions of spiral galaxies, the mean ages of the outer regions are known to be somewhat younger and more metal-poor (Bell and de Jong 2000). We can therefore expect the contribution from intermediate-age (0.6 Gyr - 2 Gyr) stars to be stronger in these outer regions, and the near-IR surface brightness of the outer disk will be preferentially enhanced by the presence of thermally-pulsing asymptotic giant branch (TP-AGB) stars. Although the average number of carbon stars per intermediate age cluster in the Magellanic clouds is only about 2.5 (Persson et al. 1983), they radiate such an enormous amount of light in the near-infrared that they contribute about 50 percent of the bolometric luminosity of the entire cluster.

There is no better place to start examining the accretion of gas in the outer domains of spiral disks than in our Local Group, and we focus our attention here on the Triangulum Spiral Galaxy M33. The history of the Andromeda Galaxy M31 is discussed elsewhere (Block et al. 2006). If accretion of gas is indeed taking place in M33, and if its disk is growing from the inside outwards, then one obvious tell-tale signature would be the presence of a red intermediate age population of carbon stars in its outer disk.

In an earlier study (Block et al. 2004), we presented near-infrared images of M33 from a deep subsample of 2MASS, in which individual stars were unresolved. The deep 2MASS images revealed remarkable arcs of red stars in the outer disk; the northern arc subtends $120^{\circ}$ in azimuth angle and $\sim 5^{\prime}$ in width (see Figure 1 ). The northern arc is dominant although a very faint southern counterpart arc, forming a partial ring, can also be seen. The arcs lie at a radius of $2-3$ disk scale lengths (in $\mathrm{V}$, the disk scale length is 0.1 degree; Ferguson et al. 2006). Of course we are not claiming that carbon stars lie only in the two outer arcs, but we do claim that a combination of increased number density, perhaps aided by an increase in the IR integrated-light contribution of carbon stars relative to the underlying stellar population due to a metallicity gradient, makes them stand out in the infrared. That there is an increase in number density at the appropriate radius is confirmed by Rowe et al. (2005).

Fourier analysis of the light distribution shows that the dominant $m=2$ peak corresponds to the giant arcs. The pitch angle of the dominant $m=2$ structure is $\sim 58$ degrees. It is these outer arcs, not the inner two-armed structure, which dominate the $m=2$ Fourier spectra at the NIR.

\section{Observations}

Table 1 lists the magnitudes and colors of 7 candidate targets in the outer northern arc. In deriving the absolute magnitudes in Table 1, we assume a distance modulus to M33 of $24.64^{\mathrm{m}}$, corresponding to a linear distance of $840 \mathrm{kpc}$ (Freedman et al. 1991). Noting that $\mathrm{E}(\mathrm{B}-\mathrm{V})=0.3 \pm 0.1 \mathrm{mag}$ (Wilson 1991), and that the K-band extinction is 


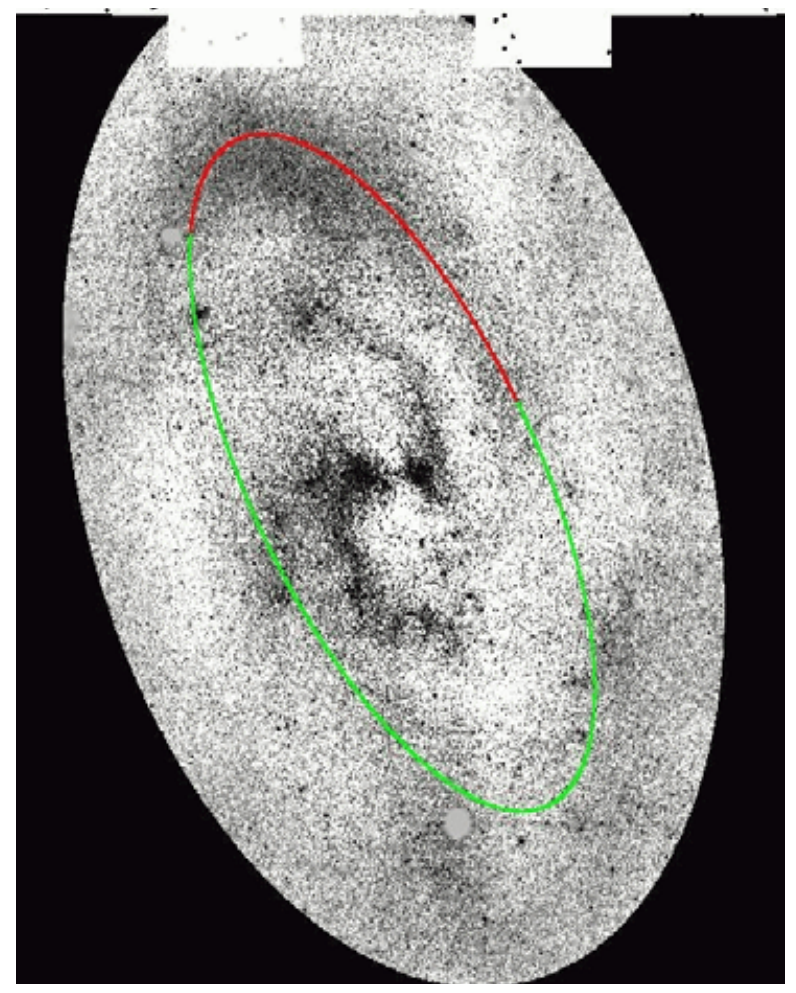

Figure 1. A partial ring of very red stars is seen in this JHKs image of M33, secured from a special set of 2MASS observations wherein the integration time was increased by a factor of six. The ring is seen to full advantage with a simple ellipsoid model subtracted. The northern plume or arc spans up to $5^{\prime}$ in width and is located at a radius of 2 to 3 disk scale lengths. An ellipsoidal swath is selected to pass through the arcs. North is up and East to the left.

Table 1. Magnitudes and colors of our target stars in the northern red arc of M33

\begin{tabular}{cccccccc}
\hline Star & $\mathrm{J}$ & $\mathrm{H}$ & $\mathrm{Ks}$ & $\mathrm{J}-\mathrm{Ks}$ & $\mathrm{J}-\mathrm{H}$ & $\mathrm{H}-\mathrm{Ks}$ & $\mathrm{M}_{\mathrm{Ks}}$ \\
\hline 1 & 17.96 & 16.76 & 15.91 & 2.05 & 1.20 & 0.85 & -8.81 \\
2 & 18.49 & 17.09 & 16.32 & 2.16 & 1.40 & 0.77 & -8.39 \\
3 & 19.61 & 18.04 & 16.66 & 2.96 & 1.57 & 1.39 & -8.06 \\
4 & 18.57 & 17.26 & 16.49 & 2.08 & 1.31 & 0.77 & -8.23 \\
5 & 18.82 & 17.35 & 16.54 & 2.28 & 1.47 & 0.81 & -8.17 \\
6 & 18.42 & 17.24 & 16.46 & 1.97 & 1.18 & 0.78 & -8.25 \\
7 & 18.37 & 16.91 & 15.82 & 2.55 & 1.46 & 1.10 & -8.89 \\
\hline
\end{tabular}

approximately one-tenth that in the optical, we use a dust extinction correction for the Ks apparent magnitudes of 0.09 mag.

Spectra of our carbon star candidates were obtained on 2004 Aug 17 UT with the Low Resolution Imaging Spectrograph (LRIS; Oke et al. 1995) attached to the Keck I telescope.

Observations of M33 were also made using all four bands of the Infrared Array Camera (IRAC) on 2005 January 21 as part of a Spitzer Guaranteed Time Observing Program (Program ID 5) conducted by Spitzer Science Working Group member R. D. Gehrz. The IRAC instrument (Fazio et al. 2004) is composed of four detectors which operate at 3.6, 


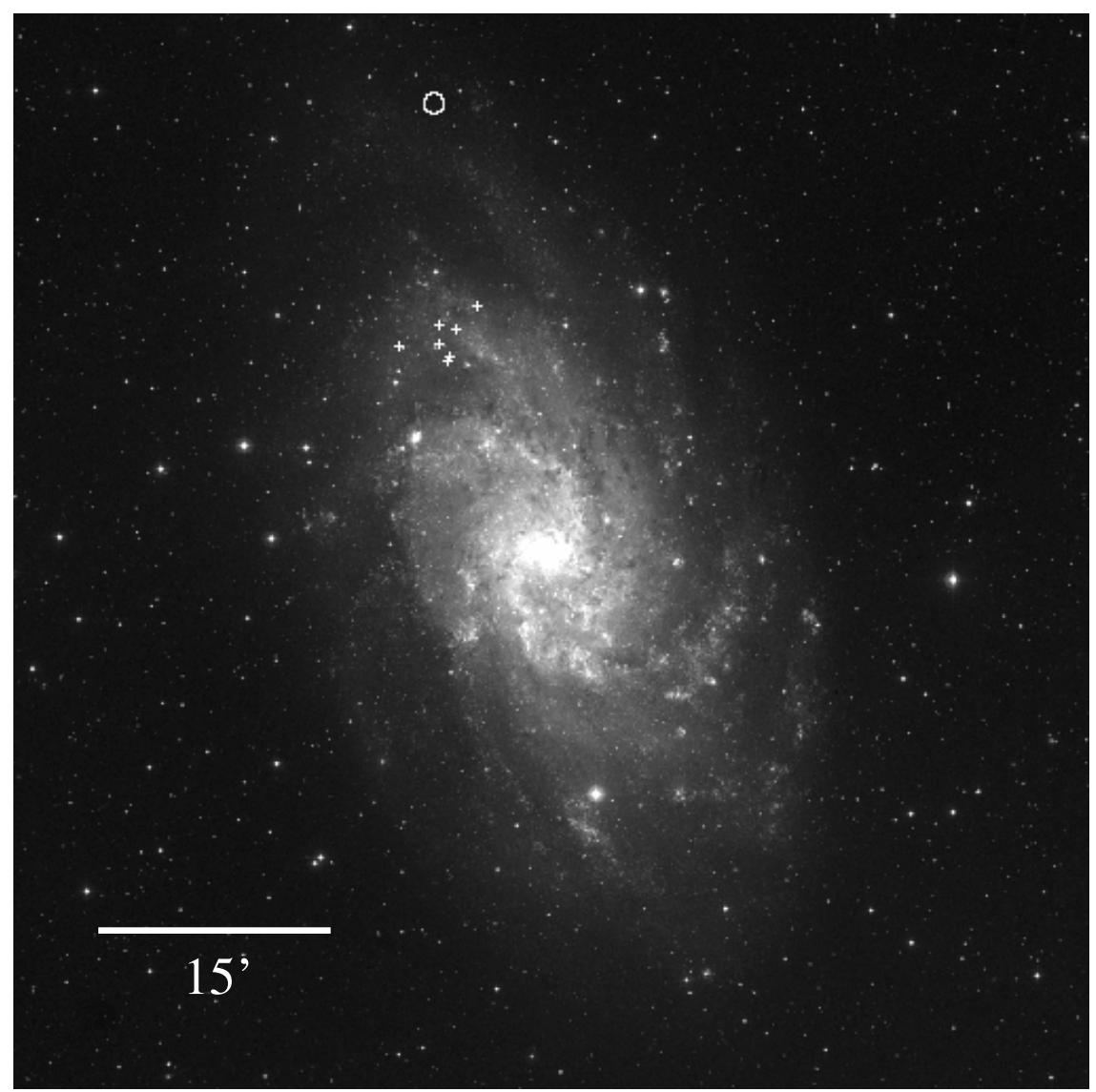

Figure 2. The positions of the seven stars listed in Table 1 are indicated by plus signs; the stars lie in the northern swath of carbon bearing stars identified photometrically in an earlier study (Block et al. 2004). Spectra of these stars (see Fig. 3) were secured using the Keck I telescope in Hawaii. Also observed (but with its twin sister telescope, Keck II) are two stars which lie 0.5 degrees away from the centre of $\mathrm{M} 33$, in an area identified by a white circle. These outlying stars are representative of a very red family identified in near-infrared imaging with the Hale $5 \mathrm{~m}$ reflector at Mount Palomar.

$4.5,5.8$, and $8.0 \mu \mathrm{m}$. All four detector arrays are 256x256 pixels in size with mean pixel scales of $1.221,1.213,1.222$, and $1.220^{\prime \prime}$ pixel $^{-1}$ respectively.

\section{Discussion and Conclusions}

Carbon stars show a plethora of molecular spectral features, including the $\mathrm{C}_{2}$ Swan bands and the CN bands. All stars in Table 1 as well as those imaged within the white circle (Figure 2) are spectroscopically confirmed to be carbon stars.

Figure 4 shows a PAH emissitivity radial profile in M33, generated from a scaled non-stellar IRAC $8 \mu m-3.6 \mu m$ image. We find a dramatic change of phase in the PAH distribution at precisely the same radius where Corbelli and Schneider (1997) find a warp in HI. Galactic warps and infall of gas are inextricably linked, as reviewed by Binney (1992).

The Keck spectra offers unique insight into how the disks of one of our closest spiral galaxies, M33, has evolved with time: it continues to grow by accretion of low-metallicity 

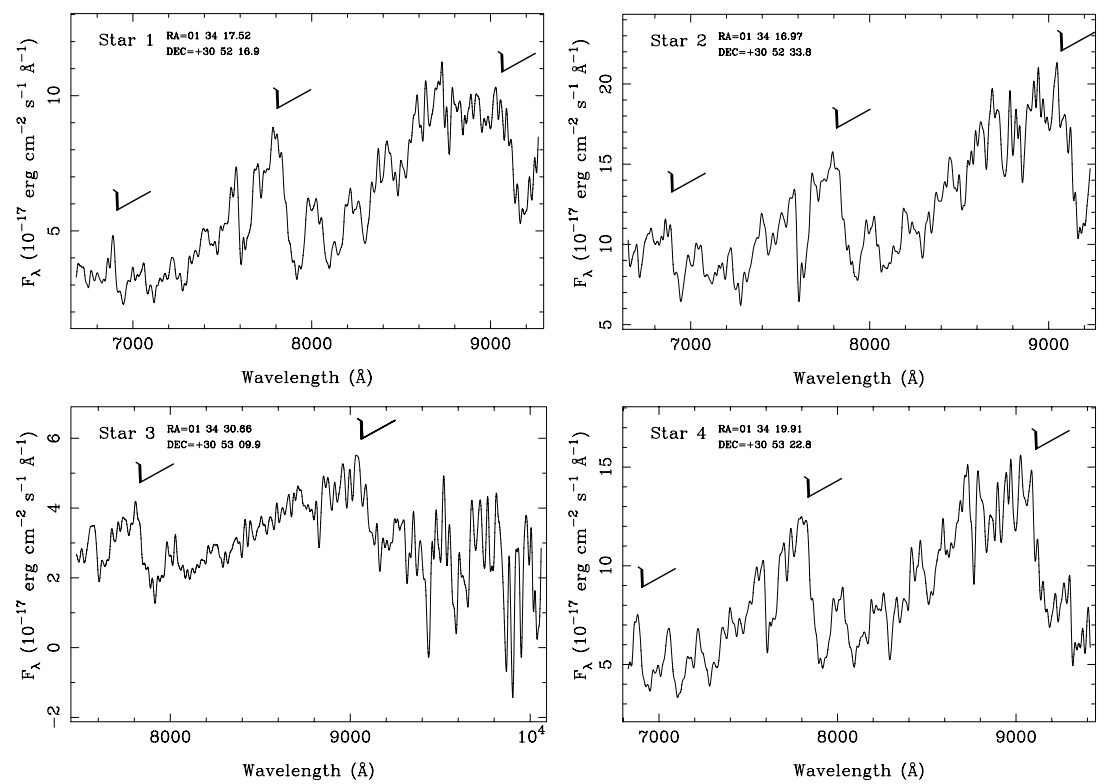

Figure 3. Spectra of four stars in the northern swath seen in Figure 1, secured using the Keck I telescope. The coordinates of each star are indicated in each panel. Carbon stars show a plethora of molecular spectral features, including the $\mathrm{C}_{2}$ Swan bands and the $\mathrm{CN}$ bands. The presence of the hugely dominant CN bands shortward of $7000 \AA$ and $8000 \AA$ and longward of $9000 \AA$ are indicated by tick marks and unambiguously reveal their C-star status.

gas from the inside, outwards. The implications of the presence of carbon stars in the outer disk of M33 immediately beckons the question of a possible ubiquity of such an intermediate age population in the outer domains of other spiral disks. At hand is the potential to exploit our technique of identifying thermally-pulsing asymptotic giant branch carbon stars in the outer domains of more distant spirals on the basis of their nearinfrared colours.

\section{Acknowledgements}

The Anglo American Cosmic Dust Laboratory is funded by the Anglo American Chairman's Fund. IP acknowledges support from the Mexican foundation CONACyT under project 35947-E. The paper is based in part on data obtained at the W. M. Keck Observatory. The Spitzer Space Telescope is operated by the JPL, Caltech under a contract with NASA. RDG and CEW were supported by NASA through an award issued by JPL/Caltech.

\section{References}

Bell, E.F., de Jong, R.S. 2000, MNRAS 312, 497

Binney, J. Ann. Rev. Astron. Astrophys. 1992, 30, 51

Block, D.L., Bournaud, F., Combes, F., Puerari, I., \& Buta, R. 2002, A\&A 394, L35

Block, D.L., Freeman, K.C., Jarrett, T.H., Puerari, I., Worthey, G., Combes, F., \& Groess, R. 2004, A\&A 425, L37

Block, D.L., Bournaud, F., Combes, F., Groess, R., Barmby, P., Ashby, M.L.N., Fazio, G.G., Pahre, M.A., \& Willner, S.P. 2006, Nature, 443, 832

Corbelli, E., \& Schneider, S.E. 1997, ApJ 479, 244

Fazio, G.G., Hora, J.L., \& Allen, L.E. et al. 2004, ApJS 154, 10 

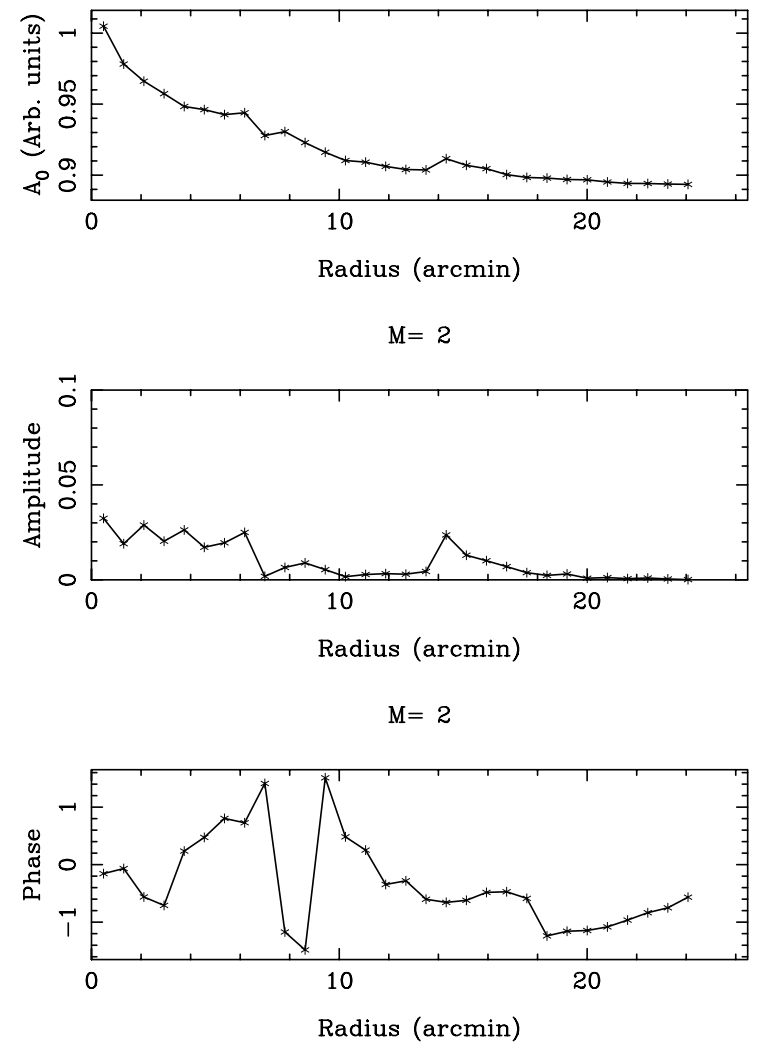

Figure 4. The topmost panel shows a PAH emissitivity radial profile. In the middle panel is shown the amplitude of the Fourier $\mathrm{m}=2$ mode as a function of radius. The local peak at $r=14^{\prime}$ coincides with the inner radial limit of the arcs. The phase of the $\mathrm{m}=2$ component is presented in the lower panel. We see a dramatic change of phase in the PAH distribution at precisely the same radius $r=18^{\prime}$ where Corbelli and Schneider (1997) find a warp in HI.

Ferguson, A., Irwin, M., Chapman, S., Ibata, R., Lewis, G., \& Tanvir, N. 2006, astro-ph/0601121 Freedman, W.L., Wilson, C.D., \& Madore, B.F. 1991, ApJ 372, 455

Keres, D., Katz, N., Weinberg, D.H., \& Davé, R. 2005 MNRAS 363, 2

Oke, J.B., Cohen, J.G., Carr, M., Cromer, J., Dingizian, A., Harris, F.H., Labrecque, S., Lucinio, R., Schaal, W., Epps, H., \& Miller, J. 1995, PASP 107, 375

Persson, S.E. Aaronson, M. Cohen, J.G. Frogel, J.A., \& Matthews, K. 1983, ApJ 266, 105

Rowe, J.F., Richer, H.B., Brewer, J.P., \& Crabtree, D.R. 2005, AJ 129, 729

Sancisi, R. 1983, Internal Kinematics and Dynamics of Galaxies, IAU Symp. 100, 55

Searle, L. 1971, ApJ 168, 327

Wilson, C.D. 1991, AJ 101, 1663

\section{Discussion}

H. RicheR: A paper recently published (Rowe et al. 2005) showed NO evidence of carbon star arcs. This was done with a 4 band photometric system which would distinguish the spectral signature of carbon stars. No giant arcs were found.

D. BLOCK: Of course we are not claiming that carbon stars lie *only* in the two outer arcs, but we do claim that a combination of increased number density, perhaps aided by an increase in the IR integrated-light contribution of carbon stars relative to the underlying stellar population due to a metallicity gradient, makes them stand out in 
the infrared. That there is an increase in number density at the appropriate radius is confirmed by Rowe et al. (2005).

When using your four filter technique (V,I, 77, 81) to detect carbon stars \& plot the number as a function of radius, there is a local peak at a radius of approximately 20 arcmin. That is precisely where the deep 2MASS images show arcs of very red stars whose color lie cooler of J-K i 1.3. In my talk, you have seen some sample Keck I and II spectra confirming the carbon star status of these very red stars.

In summary, I strongly believe that you DO see the arcs of carbon stars reported in our A\&A LETTER - these betray themselves as a local peak in your number density count. Furthermore, in the Rowe et al. paper, you mention that the arcs of carbon stars found by us do not lie outside the disk-we fully agree. In fact, the arcs lie at 2-3 disk scalengths and are fully part of the (outer) disk of the Triangulum Spiral Galaxy.

B Elmegreen: Your observation nicely explains a puzzle Debbie and I found for the UDF spirals, 280 of them, that the disk scale length increases over time, not just the overall disk size, and that really requires new matter added to the outer parts exclusively, as with your carbon stars. Then how can this far outer accretion end up in the center to rejuvenate a bar?

D. BLOCK: We indeed believe that the Triangulum Spiral Galaxy is a Rosetta Stone in this regard: it enables the disk building process to be beautifully observed at close quarters, right within our Local Group. New matter is being added to the outer parts of M33, exactly as has been observed by Debbie and yourself in the UDF spirals. With regard to the latter part of your question, several possibilities spring to mind for the outer low metallicity gas to progressively flow inward: one of these may be gravity torques associated with an outer spiral wave in M33. The wave would have a low pattern speed, placing corotation in the outer parts of M33. Other possibilities could invoke shear, viscous torques and dynamical friction.

M-M. MacLow: Why is evidence of turbulence (5/3 to 2 power spectra) able to be interpreted as evidence for accretion? Uniform accretion streaming is, of course, not turbulence.

D. BLOCK: Of course you are correct. Let me just add that the carbon star arcs are associated with the M33 HI warp, and warps and gaseous infall are inextricably linked, as reviewed by James Binney some years back. I'd be surprised if gas streaming at the locale of the warp was uniform. While that is an open question, our conclusions for M33 accreting gas are based on the presence of arcs of TP-AGB stars at the HI warp and not on the Kolmogorov or shock-dominated Burgers turbulence slopes. You are correct in saying that the PAH power spectra by themselves (generated from IRAC/Spitzer Space Telescope images at 8 microns) do not serve as a diagnostic for gas accretion. In contrast, t he Keck I and Keck II spectra strongly argue our case, wherein the Triangulum Spiral continues to accrete low metallicity gas flowing inward at the HI warp, yielding the intermediate age TP-AGB carbon stars. 